\title{
Óleo de dendê (Elaeais guineensis Jaquim) na alimentação de poedeiras leves
}

\author{
Palm oil (Elaeais guineensis Jaquim) in the diet of laying hens
}

Marina de Almeida Nogueira[a], Frank George Guimarães Cruz ${ }^{[b]}$, Ewerton de Souza Tanaka ${ }^{[c]}$, João Paulo Ferreira Rufino[d], Thiago Macedo Santana $a^{[e]}$

[a] Acadêmica do curso de Zootecnia da Universidade Federal do Amazonas (UFAM), Manaus, AM - Brasil, e-mail: nogueira. pequena@hotmail.com.br

[b] Engenheiro agrônomo, doutor em Biotecnologia, professor associado da Universidade Federal do Amazonas (UFAM), Manaus, AM - Brasil, e-mail: frankcruz@ufam.edu.br

[c] Acadêmico do curso de Zootecnia da Universidade Federal do Amazonas (UFAM), Manaus, AM - Brasil, e-mail: ewerton_ tanaka@hotmail.com.br

[d] Acadêmico do curso de Zootecnia da Universidade Federal do Amazonas (UFAM), Manaus, AM - Brasil, e-mail: joaopaulorufino@live.com.br

[e] Acadêmico do curso de Zootecnia da Universidade Federal do Amazonas (UFAM), Manaus, AM - Brasil, e-mail: thi_03@ hotmail.com

\section{Resumo}

Objetivou-se com este trabalho avaliar o desempenho zootécnico da suplementação de níveis crescentes de óleo de dendê $(0,1,2,3,4$ e 5\%) em rações para poedeiras leves. 0 experimento teve duração de 105 dias com cinco intervalos de 21 dias cada. Foram utilizadas 144 poedeiras Hissex White com 32 semanas de idade alojadas em 24 gaiolas. 0 delineamento experimental foi inteiramente casualizado com seis tratamentos e quatro repetições de seis aves cada, e os dados foram analisados utilizando regressão polinomial. 0 óleo de dendê foi utilizado nas rações como fonte energética e pigmentante da gema do ovo. 0 consumo de ração, a produção de ovos, a conversão alimentar por dúzia de ovos e a conversão alimentar por massa de ovos, não foram influenciados significativamente $(\mathrm{P}>0,05)$ pelos tratamentos. A altura do albúmen apresentou efeito quadrático, obtendo seu melhor desempenho nos níveis $2 \%$ e $5 \%$ de óleo de dendê nas rações. A espessura da casca foi afetada pelos tratamentos $(\mathrm{P}<0,05)$, tendo o nível $5 \%$ apresentado melhor resultado. A pigmentação da gema foi influenciada pelos tratamentos $(\mathrm{P}<0,05)$ aumentando proporcionalmente ao nível de inclusão de óleo de dendê nas rações, tendo o nível máximo de inclusão apresentado maior pigmentação $(6,2)$. É possível utilizar até 5\% de óleo de dendê em rações para poedeiras leves sem afetar o desempenho 
produtivo das aves, o que viabiliza o óleo de dendê como alimento alternativo a ser utilizado em inclusão nas rações das aves e fonte natural de pigmento da gema do ovo.

Palavras-chave: Desempenho. Pigmentação da gema. Inclusão.

\section{Abstract}

The objective of this study was to evaluate the zootechnic performance of increasing levels of palm oil supplementation $(0,1,2,3,4$ and 5\%) in the diet of laying hens. The experiment lasted 105 days with five intervals of 21 days. One hundred and forty four Hissex White birds with 32 weeks of age were used. The animals were distributed in 24 cages. The experimental design was a completely randomized with six treatments and four replicates of six birds each. The data was analyzed by polynomial regression. Palm oil was used as an energy source to the diet and to improve egg yolk pigmentation. Feed intake, egg production, feed conversion per dozen eggs and feed conversion per egg mass were not significantly influenced $(P>0.05)$ by the treatments. The albumen height showed a quadratic effect, achieving its best performance at levels of $2 \%$ and $5 \%$ palm oil in the diet. The shell thickness was affected by the treatments $(P<0.05)$, where the level $5 \%$ presented the best results. The yolk pigmentation was affected by the treatments $(P<0.05)$, increasing proportionally to the level of palm oil inclusion in the diet. This work showed that it is possible to use up to 5\% palm oil in the diet of laying hens without affecting production performance, enabling the use of palm oil as an alternate energy component in the diet of these animals, as well as a natural source for egg yolk's pigmentation.

Keywords: Performance. Yolk pigmentation. Inclusion.

\section{Introdução}

As formulações de dietas para aves, no contexto atual, são basicamente compostas por dois ou três ingredientes que compõem $75 \%$ do seu total, sendo os mais utilizados como fonte proteica o farelo de soja e, como fonte energética, o milho (COUSINS, 1999; PUCCI et al., 2003). Constantemente, porém, pesquisadores buscam por alimentos que possuam potencial de substituição para, na maioria dos casos, milho, farelo de soja ou aditivos que possam atuar de maneira complementar na alimentação das aves e, ao mesmo tempo, diminuam seu preço e auxiliem na maximização dos lucros.

Como aditivos, os óleos vegetais tornam-se interessantes do ponto de vista alimentar para as aves, uma vez que a sua utilização incrementa a energia nas rações, melhora a palatabilidade e facilita a digestão e absorção de constituintes não lipídicos nos ingredientes. Os óleos vegetais são importantes fontes de ácidos graxos insaturados e devem ser fornecidos via ração para permitir uma adequada nutrição e produção dos animais. A partir de 1980, as rações avícolas brasileiras passaram a usar mais frequentemente gorduras suplementares, aumentando o seu valor energético e, consequentemente, o desempenho das aves (SANTOS et al., 2009).

0 óleo de dendê (também denominado óleo de palma) é extraído a partir do processamento do fruto do dendezeiro (Elaeais guineensis Jaquim), uma palmeira originária da costa oriental da África (Golfo da Guiné) e encontrada em povoamentos subespontâneos, desde o Senegal até a Angola (SOUZA, 2000).

Devido ao seu baixo custo de produção e por ser uma das lavouras que mais crescem no mundo, o óleo de dendê é requerido como matéria-prima para diferentes segmentos nas indústrias oleoquímicas, farmacêuticas, de sabões e cosméticos. Sua principal utilização é na alimentação humana, estando presente na margarina, em gorduras sólidas, no óleo de cozinha, na maionese, na panificação, no leite, em chocolates artificiais e em frituras industriais, saltando de 17 milhões de toneladas 
produzidas em 1998 para 45 milhões de toneladas em 2010 (PROJETO BIODIESEL, 2007; BECKER, 2011).

O Brasil possui o maior potencial mundial para a produção do óleo de dendê, em virtude dos cerca de 75 milhões de hectares de terras aptas à dendeicultura. A Amazônia possui cerca de 70 milhões de hectares considerados aptos ou aproveitáveis para o cultivo do dendezeiro, sendo o Amazonas o estado que possui a maior área potencial para o plantio do dendê - cerca de 50 milhões de hectares, correspondendo a $71,4 \%$ do total (SUFRAMA, 2006). Desta potencial área presente na Amazônia, somente 39 mil hectares são utilizados efetivamente para a cultura, sendo que quase $85 \%$ dessa área cultivada estão localizadas no estado do Pará.

0 aumento da produção de óleo de dendê no Brasil deu-se, além das diversas aplicações, pelo incentivo lançado pelo Governo Federal no Programa Nacional de Produção e de Uso de Biodiesel Produção Sustentável de Óleo de Palma, gerando energia (biocombustível) e, ao mesmo tempo, garantindo a preservação ambiental (MDA, 2011). Através destas ações, a dendeicultura, hoje, constitui uma das mais rentáveis fontes de renda a serem exploradas no agronegócio da Amazônia.

Segundo Miranda e Moura (2003), o óleo de dendê é formado de triglicerídeos que são predominantemente ésteres, produtos da condensação entre glicerol e ácidos graxos. Aproximadamente $98 \%$ do óleo de dendê bruto é composto pelos seguintes ácidos graxos: saturados (32 a $45 \%$ de palmítico e de 2 a $7 \%$ de esteárico) e insaturados (38 a $52 \%$ de oleico e 5 a $11 \%$ de linoleico). Conforme o levantamento de Abdalla et al. (2008) há, ainda, vários outros coprodutos do biodiesel. Dentre eles, a torta de dendê, caracterizada por $14-15 \%$ de proteína bruta (PB), 6 a $7 \%$ de extrato etéreo (EE) e 38 a $43 \%$ de fibra bruta (FB).

Brandão (2008) afirma que as exigências de lipídeos nas dietas estão relacionadas à necessidade das aves de obter ácidos graxos que não podem ser sintetizados no organismo. 0 alto valor energético e a alta digestibilidade influenciam positivamente no desempenho das aves. A partir dessa constatação, a utilização de óleos vegetais como fonte de ácidos graxos, apresenta algumas vantagens em relação à gordura animal, rica em ácidos graxos saturados. Os óleos ricos em ácidos graxos insaturados são absorvidos mais facilmente e, por isso, apresentam valores mais altos de energia metabolizável, promovendo melhor desempenho das aves (GAIOTTO et al., 2000).

Em razão da alta capacidade de produção do dendê na região Amazônica e do alto valor energético de seu óleo, realizou-se este trabalho com o objetivo de avaliar o desempenho zootécnico da inclusão do óleo de dendê, em níveis crescentes, em rações de poedeiras comerciais leves, além de funcionar como um estímulo a sua produção .

\section{Materiais e métodos}

Oexperimento foi realizado no Setor de Avicultura do Departamento de Produção Animal e Vegetal (DPAV) da Faculdade de Ciências Agrárias (FCA) da Universidade Federal do Amazonas (UFAM), localizado no Setor Sul do Campus Universitário, em Manaus (AM), com coordenadas geográficas de latitude $3^{\circ} 06^{\prime} 14^{\prime \prime}$, longitude 59 58' 46" W. Conforme a classificação proposta por Köppen, o clima é classificado como tropical quente e úmido, com precipitação média anual de $2.286 \mathrm{~mm}$ e temperatura média variando entre 27 a $29^{\circ} \mathrm{C}$ (INMET, 2011).

0 período experimental compreendeu de fevereiro a junho de 2013, com cinco intervalos de 21 dias. Anteriormente ,entretanto, as aves foram submetidas a um período de 15 dias de adaptação.

0 experimento foi realizado em galpão medindo $17 \mathrm{~m}$ de comprimento e 3,5 m de largura, possuindo gaiolas de arame galvanizado, comedouros tipo calha e bebedouros tipo niple. Foram utilizadas 144 poedeiras da linhagem Hissex White com 32 semanas de idade, alojadas em gaiolas medindo 1,00 x 0,40 x 0,45 m e criadas sob idênticas condições de alimentação e manejo. Durante todo o período experimental foram fornecidas 16 horas de luz/dia (natural + artificial). Todas as aves foram pesadas no início do experimento para uniformização das parcelas, apresentando peso médio de 1,36 $\pm 0,082$ $\mathrm{kg}$. A coleta de ovos foi realizada três vezes ao dia (às 8,11 e 16h).

As aves foram distribuídas em um delineamento experimental inteiramente casualizado (DIC), constituído de seis tratamentos $(0,1,2,3,4$ e 5\% de suplementação de óleo de dendê nas rações) e quatro repetições de seis aves cada. 
0 óleo de dendê utilizado foi obtido junto ao Centro de Pesquisa Agropecuária da Amazônia (CPAA - EMBRAPA), sendo o produto extraído a partir do fruto do dendezeiro por processo de prensagem, obedecendo às seguintes etapas: esterilização, em que o cacho com os frutos do dendezeiro foi submetido ao vapor d'água a $130{ }^{\circ} \mathrm{C}$ e $2 \mathrm{~kg} / \mathrm{cm}^{2}$ de pressão por 60 minutos, evitando o desenvolvimento da acidez e facilitando o desprendimento do fruto; debulha do cacho, onde o mesmo foi levado ao debulhador para a separação dos frutos que, em seguida, passaram pelo digestor para a liberação do óleo das células oleíferas. A massa liberada pelo digestor foi prensada e o óleo de dendê extraído. A torta (sementes + fibras) passou pelo desfibrador para a liberação das sementes, sendo estas levadas ao polidor para a eliminação de todo o resíduo de fibra ainda presente e, após, ao secador. Por fim, passaram pela descascadora centrífuga onde foram quebradas, separando-se as cascas das amêndoas. As amêndoas então foram trituradas e prensadas para a liberação do óleo e da torta de palmíste. Todo o óleo obtido do processo foi depurado e armazenado em tanques para sua melhor conservação.

As exigências nutricionais das aves foram atendidas conforme o manual da linhagem utilizada (GLOBOAVES, 2006). As rações isonutritivas foram formuladas de acordo com os valores dos ingredientes fornecidos pelas Tabelas Brasileiras para Aves e Suínos (ROSTAGNO, 2011), com exceção da composição do óleo de dendê, e encontram-se na Tabela 1.

No desempenho das aves foram avaliadas as variáveis: consumo de ração (g/ave/dia), produção de ovos (\%), conversão alimentar por massa de ovos (kg de ração/ kg de massa de ovo), conversão alimentar por dúzia de ovos (kg de ração/dúzia de

Tabela 1 - Composição das rações experimentais contendo óleo de dendê

\begin{tabular}{|c|c|c|c|c|c|c|}
\hline \multirow[b]{3}{*}{ Ingredientes } & \multicolumn{6}{|c|}{ Nível de óleo de dendê (\%) } \\
\hline & 0 & 1 & 2 & 3 & 4 & 5 \\
\hline & 1 & II & III & IV & V & VI \\
\hline Milho & 62,80 & 60,62 & 59,39 & 58,16 & 56,93 & 55,70 \\
\hline Farelo Soja & 24,25 & 25,82 & 26,05 & 26,27 & 26,50 & 26,73 \\
\hline Calcário & 8,68 & 9,20 & 9,20 & 9,19 & 9,19 & 9,18 \\
\hline Fosfato bicálcico & 2,39 & 2,39 & 2,39 & 2,41 & 2,41 & 2,41 \\
\hline Óleo de dendê & 0,00 & 1,00 & 2,00 & 3,00 & 4,00 & 5,00 \\
\hline Premix Vit. Min. ${ }^{1}$ & 0,50 & 0,50 & 0,50 & 0,50 & 0,50 & 0,50 \\
\hline Sal & 0,35 & 0,35 & 0,35 & 0,35 & 0,35 & 0,35 \\
\hline DL- Met 99 & 1,03 & 0,11 & 0,11 & 0,11 & 0,11 & 0,12 \\
\hline Butil-Hidroxi-Tolueno $(\mathrm{BHT})^{2}$ & 0,00 & 0,01 & 0,01 & 0,01 & 0,01 & 0,01 \\
\hline Total (Kg) & 100,00 & 100,00 & 100,00 & 100,00 & 100,00 & 100,00 \\
\hline \multicolumn{7}{|l|}{ Nutriente calculado } \\
\hline Energia Metabo., $\mathrm{kcal}^{-1} / \mathrm{kg}$ & 2,750 & 2,754 & 2,805 & 2,856 & 2,907 & 2,958 \\
\hline Proteína bruta, \% & 17,000 & 17,000 & 17,000 & 17,000 & 17,000 & 17,000 \\
\hline Metionina + Cistina, \% & 1,555 & 0,670 & 0,670 & 0,670 & 0,656 & 0,656 \\
\hline Metionina, \% & 1,274 & 0,383 & 0,383 & 0,384 & 0,385 & 0,385 \\
\hline Cálcio, \% & 4,000 & 4,200 & 4,200 & 4,200 & 4,200 & 4,200 \\
\hline Fósforo disponível, \% & 0,450 & 0,450 & 0,450 & 0,450 & 0,450 & 0,450 \\
\hline Sódio, \% & 0,174 & 0,175 & 0,175 & 0,175 & 0,175 & 0,175 \\
\hline
\end{tabular}

Nota: *1 Níveis de garantia por quilograma de produto: Vitamina A 2.000.000 UI, Vitamina D3 400.000 UI, Vitamina E 2.400 mg, Vitamina K3 400 mg, Vitamina B1 100 mg, Vitamina B2 760 mg, Vitamina B6 100 mg, Vitamina B12 2.400 mcg, Niacina 5.000 mg, Pantotenato de Cálcio 2000 mg, Ácido Fólico 50 mg, Cocciostático 12.000 mg, Colina 50.000 mg, Cobre 1.200 mg, Ferro 6.000 mg, Manganês 14.000 mg, Zinco 10.000 mg, lodo 100 mg. Selênio 40 mg. Veículo Q.S.P. 1.000 g. ${ }^{* 2}$ Antioxidante. Fonte: Dados da pesquisa. 
ovo) e a massa de ovo (g). Nos dois últimos dias de cada período de avaliação (21 dias), foram coletados 24 ovos de cada tratamento (quatro ovos de cada repetição) para análise da qualidade, onde foram avaliados o peso do ovo (g), o peso do albúmen (g), o peso da gema (g), a altura do albúmen $(\mathrm{mm})$, a altura da gema $(\mathrm{mm})$, o peso da casca $(\mathrm{g})$, a espessura da casca $(\mu \mathrm{m})$, a gravidade específica $\left(\mathrm{g} / \mathrm{cm}^{3}\right)$ e a pigmentação da gema. Antes de serem submetidos à avaliação, os ovos coletados foram armazenados durante uma hora, a fim de sua temperatura se equiparar à temperatura ambiente.

Os ovos foram pesados em balança eletrônica com aproximação de $0,01 \mathrm{~g}$, sendo considerado o peso em gramas dos ovos inteiros com casca. A massa de ovo foi obtida através do cálculo do quociente entre peso do ovo e a produção de ovos multiplicada por cem.

Logo após a pesagem, os ovos inteiros foram colocados em cestas de arame e imersos em baldes plásticos contendo diferentes soluções de cloreto de sódio $(\mathrm{NaCl})$, da menor para a maior concentração, com variações de densidade que foram de 1,075 a $1,100 \mathrm{~g} / \mathrm{cm}^{3}$ e intervalo de 0,005 entre elas. Antes de seguirem para as soluções salinas, os ovos passaram por um balde contendo água pura e foram retirados ao flutuarem até a superfície, anotando-se seus respectivos valores.

Para a análise de peso do albúmen e da gema, os mesmos foram separados por um separador manual, colocados apartadamente em copos plásticos (de peso previamente tirado em balança analítica) e pesados. Para os cálculos de altura, foram colocados, após serem pesados, sobre uma placa plana para mensuração de seus respectivos valores. 0 procedimento para a determinação da altura consiste em medir na região mediana, entre a borda externa do albúmen e a gema. A mensuração se deu com a utilização de um paquímetro eletrônico, sendo os valores anotados em milímetros. Os pesos das cascas dos ovos foram obtidos após as mesmas serem lavadas, secadas à temperatura ambiente por 48 horas e, posteriormente, pesadas em gramas.

Para a determinação da espessura da casca, foram utilizadas as cascas secas anteriormente pesadas, tendo a medição sido feita com o auxílio de um micrômetro. As leituras foram efetuadas em três pontos da casca: na região basal, equatorial e apical, anotando-se os valores obtidos. A partir destes, calculou-se a média, em micrometro, da espessura das casca dos ovos. Para avaliação da pigmentação da gema dos ovos, utilizou-se o leque colorimétrico Roche com pontos de 1 a 15. Para determinação da unidade Haugh, utilizou-se a fórmula proposta por Silva (2004).

A análise estatística foi realizada pelo programa computacional Statistical Analysis System (SAS, 2008), e as estimativas dos tratamentos foram submetidas à análise de regressão polinomial.

\section{Resultados}

Os resultados médios obtidos para as variáveis relacionadas ao desempenho das aves estão expostos na Tabela 2.

Tabela 2 - Consumo de ração, conversão alimentar por dúzia de ovos (kg/dz), conversão alimentar por massa de ovos $(\mathrm{kg} / \mathrm{kg})$, percentagem de postura e massa de ovos de poedeiras comerciais alimentadas com rações contendo diferentes níveis óleo de dendê

\begin{tabular}{|c|c|c|c|c|c|c|c|}
\hline \multirow{2}{*}{ Variáveis* } & \multicolumn{6}{|c|}{ Níveis de inclusão de óleo de dendê (\%) } & \multirow{2}{*}{$\begin{array}{c}\mathrm{CV} \\
\%\end{array}$} \\
\hline & 0 & 1 & 2 & 3 & 4 & 5 & \\
\hline Consumo de ração (g/ave/dia) & 112,57 & 111,50 & 116,54 & 107,91 & 110,98 & 109,76 & 3,50 \\
\hline Conversão alimentar (kg/dz) & 1,59 & 1,49 & 1,58 & 1,43 & 1,60 & 1,59 & 9,05 \\
\hline Conversão alimentar (kg/kg) & 2,20 & 2,04 & 2,17 & 1,97 & 2,21 & 2,19 & 8,86 \\
\hline Postura (\%) & 85,43 & 89,76 & 88,53 & 90,11 & 83,41 & 83,01 & 6,58 \\
\hline Massa de ovo (g/ave $\left.{ }^{-1} / \mathrm{dia}^{-1}\right)$ & 51,73 & 54,65 & 53,85 & 54,84 & 50,12 & 50,08 & 6,56 \\
\hline
\end{tabular}

Legenda: CV - Coeficiente de variação.

Nota: ${ }^{*}>0,05=$ não significativo.

Fonte: Dados da pesquisa. 
A Tabela 3 apresenta os resultados médios obtidos para peso do ovo, percentagem de albúmen, percentagem de gema, percentagem de casca, altura do albúmen, altura da gema, unidade Haugh, espessura da casca, gravidade específica e pigmentação da gema, variáveis relacionadas à qualidade do ovo.

\section{Discussão}

Na Tabela 2, não foi observada diferença significativa $(\mathrm{P}>0,05)$ entre os níveis de inclusão de óleo de dendê sobre o consumo de ração, havendo decréscimo no consumo à medida que aumentaram-se os níveis de inclusão, corroborando com Duarte et al. (2004) que, em pesquisa, confirmou que o excesso de energia na dieta leva o animal a satisfazer suas necessidades com menor consumo de ração.

Para a produção de ovos não foram encontradas diferenças significativas $(\mathrm{P}>0,05)$. Entretanto, o nível de inclusão de 3\% de óleo de dendê apresentou melhores resultados, corroborando com Grobas et al. (1999). À medida que a ave inicia uma ingestão exacerbada de gorduras, estas afetam diretamente na absorção dos demais nutrientes e, consequentemente, diminuem a produção devido à necessidade dos mesmos para a formação do ovo. 0 tratamento com 3\% de inclusão do óleo de dendê também apresentou melhores resultados para a conversão alimentar por dúzia e massa de ovos produzidos, discordando dos resultados obtidos por Filardi (2005) e corroborando com os resultados encontrados por Santos (2005), mas sem apresentar diferenças significativas $(\mathrm{P}>0,05)$ entre os tratamentos. Para massa de ovo tampouco foram constatadas diferenças significativas $(\mathrm{P}>0,05)$. As conversões alimentares apresentam correlação direta com o consumo de ração, sendo que, independentemente do aumento de seus níveis calóricos, as conversões mantiveram-se abaixo dos resultados da ração referência (sem inclusão de óleo). Tais resultados tornam a inclusão do óleo de dendê, de fato, um elemento atrativo ao produtor que deseja uma conversão mais baixa dos parâmetros da relação consumo-produtividade das aves sem, no entanto, afetar o desempenho das mesmas.

É importante frisar que os resultados encontrados para o desempenho das aves submetidas às rações com diferentes níveis de óleo de dendê, devem ser correlacionados com o ambiente onde estas foram manejadas. 0 estado do Amazonas localiza-se em uma região de clima tropical úmido, no qual têm-se temperaturas médias entre 35 e $38{ }^{\circ} \mathrm{C}$ e umidade relativa do ar estabilizada em faixas acima de $70 \%$, o que associado ao teor calórico elevado das rações com óleo

Tabela 3 - Médias das variáveis de qualidade do ovo de poedeiras comerciais alimentadas com rações contendo diferentes níveis de óleo de dendê

\begin{tabular}{|c|c|c|c|c|c|c|c|c|}
\hline \multirow[b]{2}{*}{ Variáveis } & \multicolumn{5}{|c|}{ Níveis de inclusão de óleo de dendê (\%) } & \multirow[b]{2}{*}{5} & \multirow{2}{*}{$\mathrm{R}^{2}$} & \multirow{2}{*}{$\mathrm{CV}, \%$} \\
\hline & 0 & 1 & 2 & 3 & 4 & & & \\
\hline Peso do ovo (g) & 60,47 & 61,03 & 60,76 & 60,85 & 60,18 & 60,43 & - & 3,80 \\
\hline Percentagem de albúmen (\%) & 56,30 & 57,06 & 57,49 & 56,77 & 57,32 & 57,31 & - & 4,33 \\
\hline Percentagem de gema (\%) & 27,34 & 26,76 & 27,32 & 27,59 & 27,89 & 27,52 & - & 3,89 \\
\hline Percentagem de casca (\%) & 9,87 & 10,07 & 9,81 & 9,99 & 10,03 & 10,15 & - & 3,69 \\
\hline Altura do albúmen* (mm) & $3,88 \mathrm{a}$ & $3,85 \mathrm{a}$ & 3,82 a & $3,91 \mathrm{a}$ & $4,22 \mathrm{a}$ & 4,19 a & 0,38 & 5,07 \\
\hline Altura da gema (mm) & 14,34 & 14,48 & 14,44 & 14,64 & 14,58 & 14,68 & - & 1,64 \\
\hline Unidade Haugh & 65,23 & 65,80 & 65,53 & 65,60 & 64,85 & 65,11 & - & 0,20 \\
\hline Espes. da casca* $0,01(\mu \mathrm{m})$ & $40,01 b$ & $40,40 \mathrm{~b}$ & 41,04 a & $41,32 \mathrm{a}$ & 41,25 a & 41,28 a & 0,83 & 0,60 \\
\hline Gravidade específica $(\mathrm{g} / \mathrm{mL})$ & 1087,00 & 1086,81 & 1086,00 & 1086,75 & 1087,06 & 1087,50 & - & 0,24 \\
\hline Pigmentação da gema* & $4 d$ & $4,71 \mathrm{c}$ & $4,81 \mathrm{c}$ & $5,5 b$ & $5,8 a b$ & $6,2 \mathrm{a}$ & 0,93 & 8,90 \\
\hline
\end{tabular}

Legenda: CV - Coeficiente de variação,

Nota: *Efeito quadrático $(P<0,05)$. Na linha, médias seguidas de letras diferentes diferem entre si, pelo teste de Tukey a $5 \%$.

Fonte: Dados da pesquisa.

Rev. Acad., Ciênc. Agrár. Ambient., Curitiba, v. 12, n. 2, p. 103-111, abr./jun., 2014 
de dendê, tende a causar estresse calórico e consequente queda no consumo de ração das aves, influenciando diretamente nos demais índices produtivos sem, porém, apresentar diferenças significativas em seus resultados.

Conforme mostra a Tabela 3, não foram encontradas diferenças significativas $(\mathrm{P}>0,05)$ no peso do ovo, percentual de gema, percentual de casca, percentual de albúmen, altura da gema, unidade Haugh e gravidade específica. Foi observado crescimento linear positivo do peso do ovo à medida que se aumentou o nível do óleo de dendê nas rações. Segundo Grobas et al. (2001), a inclusão de alimentos de origem vegetal que sejam ricos em gorduras, proporcionam aumento no peso da gema e, consequentemente, aumento no peso dos ovos.

A gravidade específica e a percentagem de casca apresentaram melhores resultados na inclusão de $5 \%$ do óleo de dendê. 0 resultado corrobora com Peebles e McDaniel (2004), que consideraram em seu trabalho o valor da gravidade específica 1,080 como o valor limite entre baixa e alta qualidade da casca dos ovos, além de afirmarem haver uma relação diretamente proporcional entre a gravidade específica e a qualidade da casca dos ovos. Tal relação está relatada por Hamilton (1982), onde o autor afirma que a gravidade específica do ovo se reduz à medida que a espessura da casca diminui, o que também reduz sua resistência à quebra.

A altura do albúmen foi influenciada significativamente $(\mathrm{P}<0,05)$ pelos tratamentos, apresentando efeito quadrático representado pela equação $Y$ $=3,92-0,084 x+0,023 x^{2} R^{2}=0,38$, onde a partir da derivação da função foi possível estimar a altura ótima de albúmen $(3,84 \mathrm{~mm})$ no nível de 1,82\% em relação aos níveis de inclusão de óleo de dendê. O nível de inclusão de 4\%, no entanto, apresentou maior altura de albúmen.

A inclusão de gorduras na ração promove uma maior concentração de lipídios absorvidos e depositados na gema do ovo, o que pode contribuir para o seu aumento. Em contrapartida, não foram encontradas diferenças significativas $(P>0,05)$ para a altura da gema. Ao se acrescentar nas rações o óleo de dendê, fonte de suplementação calórica, há uma tendência para o aumento de sua proteína com o intuito de manter o balanceamento energia-proteína, fator que afeta no volume e, consequentemente, na altura do albúmen e da gema.
Não foram encontradas diferenças significativas $(\mathrm{P}>0,05)$ para unidade Haugh, sendo o nível de $2 \%$ de inclusão de óleo de dendê na ração o que obteve melhor resultado. As médias encontradas para Unidade Haugh corroboram com as afirmações de Silva (2004), segundo as quais quanto maior o valor da UH, melhor será a qualidade dos ovos, classificando-os em tipo AA (100 até 72), A (71 até 60), B (59 até 30$)$ e C ( 29 até 0$)$.

Foi observado efeito quadrático $(\mathrm{P}<0,05)$ na inclusão do óleo de dendê para a espessura da casca, apresentando a equação $\mathrm{Y}=0,39+0,0091 \mathrm{x}$ - $0,00092 x^{2} R^{2}=0,83$. A derivação desta função resultou no ponto de melhor espessura de casca $(36,7 \mu \mathrm{m})$, ao nível $4,94 \%$ de inclusão de óleo de dendê. Brito et al. (2005), utilizando níveis crescentes de gérmen integral de milho (rico em fibra e gordura) em rações de poedeiras leves, não encontrou efeito significativo $(\mathrm{P}>0,05)$ na qualidade da casca. Os níveis crescentes de óleo de dendê, contendo altos teores de gordura, aumentaram significativamente sua espessura, discordando de Cleaver, Christensen e Ort (1986), Bohnsack et al. (2001). Os autores, contrariamente, afirmaram que o nível elevado de gordura no intestino das aves pode afetar a absorção de nutrientes, principalmente de cálcio.

A pigmentação da gema foi influenciada significativamente $(\mathrm{P}<0,05)$ pelos tratamentos, apresentando efeito quadrático positivo representado pela equação $Y=3,56+0,50 x-0,0104 x^{2} R^{2}=0,93$. 0 nível $5 \%$ de inclusão de óleo de dendê na ração promoveu a maior pigmentação na gema, enquanto a ração referência, a menor. Estes resultados corroboram com os estudos de Faitarone (2010), que verificou a influência da adição de óleos vegetais na pigmentação da gema do ovo ao avaliar diferentes níveis de óleo de linhaça, canola, soja e suas combinações em rações para aves. Segundo o autor, à medida que se aumentou a concentração de pigmentos na alimentação das aves, intensificou-se o escore de pigmentação da gema do ovo pela classificação na escala Roche. 0 óleo de dendê, devido a sua composição, destacando-se o alto teor de carotenóides presentes, contribui de forma direta no aumento do escore de classificação da pigmentação da gema do ovo, aumentando-o consideravelmente de acordo com o nível de inclusão a ser utilizado. 


\section{Conclusão}

Em rações de poedeiras leves, o óleo de dendê pode ser utilizado como alimento alternativo com potencial de inclusão e fonte natural de pigmento da gema do ovo. É possível utilizar até 5\% de óleo de dendê em rações de poedeiras sem afetar o desempenho produtivo, peso do ovo, percentagem do albúmen, percentagem de gema, percentagem de casca, altura da gema, unidade Haugh e gravidade específica.

\section{Referências}

ABDALLA, A. L. et al. Utilização de subprodutos da indústria de biodiesel na alimentação de ruminantes. Revista Brasileira de Zootecnia, v. 37, n. spe, p. 260-268, 2008. doi:10.1590/S1516-35982008001300030.

BECKER, B. K. Recuperação de áreas desflorestadas da Amazônia: será pertinente o cultivo da palma de óleo (Dendê)?. Revista Franco-Brasileira de Geografia, v. 10, n. 10, 2011. doi:10.4000/confins.6609.

BOHNSACK, C. R. et al. Performance of commercial layers when fed diets with four levels of corn oil or poultry fat. The Journal of Applied Poultry Research, v. 11, p. 6876, 2001. doi:10.1093/japr/11.1.68.

BRANDÃO, T. M. Diferentes tipos de óleos de soja e níveis de energia em dietas de frango: desempenho e características de carcaça. 2008. 62 f. Dissertação (Mestrado em Ciência Animal) - Universidade Federal do Piauí, Teresina, 2008.

BRITO, A. B. et al. Desempenho e qualidade dos ovos de poedeiras comerciais de 30 a 64 semanas de idade consumindo gérmen integral de milho. Acta Scientiarum Animal Sciences, v. 27, n. 1, p. 29-34, 2005. doi:10.4025/ actascianimsci.v27i1.1256

CLEAVER, W. T.; CHRISTENSEN, V. L.; ORT, J. F. Physiological characteristics of a molt and second cycle of egg laying in turkey breeder hens. Poultry Science, v. 65, n. 12, p. 2335-2342, 1986. doi:10.3382/ps.0652335.

COUSINS, B. Enzimas na nutrição de aves. In: SIMPÓSIO INTERNACIONAL ACAV - EMBRAPA SOBRE NUTRIÇÃO DE AVES, 1., 1999, Concórdia. Anais... Concórdia: ACAV, 1999. p. 132-118.
DUARTE, K. F. et al. Níveis de energia e programas de alimentação sobre o desempenho e características de carcaça de frangos de corte de 42 a 57 dias de idade. In: CONFERÊNCIA APINCO DE CIÊNCIA E TECNOLOGIA AVÍCOLA, 25., 2004, Santos. Anais... Campinas: Apinco, 2004. p. 29.

FAITARONE, A. B. G. Fornecimento de fontes lipídicas na dieta de poedeiras e seus efeitos sobre o desempenho, qualidade dos ovos, perfil de ácidos graxos e colesterol na gema. 2010. $108 \mathrm{f}$. Tese (Doutorado em Zootecnia) - Faculdade de Medicina Veterinária e Zootecnia, Universidade Estadual Paulista "Júlio de Mesquita Filho", Botucatu, 2010.

FILARDI, R. S. et al. Influence of different fat sources on the performance, egg quality, and lipid profile of egg yolks of commercial layers in the second laying cycle. The Journal of Applied Poultry Research, v. 14, n. 2, p. 258264, 2005. doi:10.1093/japr/14.2.258.

GAIOTTO, J. B. et al. Óleo de soja, óleo ácido de soja e sebo bovino como fontes de gorduras em rações em frangos de corte. Revista Brasileira de Ciência Avícola, v. 2, n. 3, p. 219-227, 2000. doi:10.1590/S1516-635X2000000300004.

GLOBOAVES. Manual de manejo das poedeiras Hissex White. Cascavel: Globoaves Agropecuária Ltda., 2006.

GROBAS, S. et al. Influence of source and percentage of fat added to diet on performance and fatty acid composition of egg yolks of two strains of laying hens. Poultry Science, v. 80, n. 8, p. 1171-1179, 2001. doi:10.1093/ ps/80.8.1171.

GROBAS, S. et. al. Laying hen productivity as affected by energy, supplemental fat, and linoleic acid concentration of the diet. Poultry Science, v. 78, n. 11, p. 1542-1551, 1999. doi:10.1093/ps/78.11.1542.

HAMILTON, R. M. G. Methods and factors that affect the measurement of egg shell quality. Poultry Science, v. 61, n. 10, p. 2022-2039, 1982. doi:10.3382/ps.0612022.

INSTITUTO NACIONAL DE METEOROLOGIA - INMET. Gráficos de Estações Convencionais - Manaus. Disponível em: <http://www.inmet.gov.br/portal/index.php?r=home/page\&page=rede_estacoes_conv_graf $>$. Acesso em: 12 set. 2013.

MINISTÉRIO DO DESENVOLVIMENTO AGRÁRIO - MDA. Programa Nacional de Produção e Uso de Biodiesel: inclusão social e desenvolvimento territorial. 2011. Disponível em:<http://portal.mda.gov.br/portal/saf/arquivos/view/biodisel/arquivos2011/Biodiesel_Book_final_Low_Completo.pdf>. Acesso em: 24 set. 2013. 
MIRANDA, R. M.; MOURA, R. D. Óleo de dendê, alternativa ao óleo diesel como combustível para geradores de energia em comunidade da Amazônia. In: ENCONTRO DE ENERGIA NO MEIO RURAL, 3., 2003, Campinas. Anais... Campinas: NIPE/UNICAMP, 2003.

PEEBLES, E. D.; McDANIEL, C. D. A practical manual for understanding the shell structure of broiler hatching eggs and measurements of their quality. Bulletin 1139. Starkville, MS: Office of Agricultural Communications / Mississippi State University, 2004.

PROJETO BIODIESEL. Viabilidade de extração de óleo de dendê no estado do Pará: texto para revisão e crítica. Viçosa: MDA / UFV, 2007.

PUCCI, L. E. A. et al. Níveis de óleo e adição de complexo enzimático na ração de frangos de corte. Revista Brasileira de Zootecnia, v. 32, n. 4, p. 909-917, 2003. doi:10.1590/S1516-35982003000400017.

ROSTAGNO, H. S. Tabelas brasileiras para aves e suínos: composição de alimentos e exigências nutricionais. Viçosa: Editora UFV, 2011.

SANTOS, M. S. V. Avaliação do desempenho e qualidade dos ovos de poedeiras comerciais, submetidas às dietas suplementadas com diferentes óleos vegetais. 2005. $174 \mathrm{f}$. Tese (Doutorado em Zootecnia) Universidade Federal do Ceará, Fortaleza, 2005.
SANTOS, M. S. V. et al. Desempenho e qualidade dos ovos de poedeiras comerciais submetidas às dietas com diferentes óleos vegetais. Revista Brasileira Saúde e Produção Animal, v. 10, n. 3, p 654-667, 2009.

SILVA, F. H. A. Curso teórico-prático sobre técnicas básicas de avaliação de qualidade do ovo. Piracicaba: ESALQ, 2004.

SOUZA, J. Dendê. Jornal CEPLAC Notícias. 2000. Disponível em: <http://www.ceplac.gov.br/radar/dende. htm>. Acesso em: 25 set. 2013.

STATISTICAL ANALYSIS SYSTEM - SAS. SAS/STAT: user's guide. Version 9.2. Cary, NC: SAS Institute Inc., 2008.

SUPERINTENDÊNCIA DA ZONA FRANCA DE MANAUS SUFRAMA. Potencialidades regionais: estudo de viabilidade econômica - Dendê - Sumário Executivo. v. 5. Manaus: SUFRAMA, 2003.

Recebido: 18/10/2013

Received: $10 / 18 / 2013$

Aprovado: 07/03/2014

Approved: 03/07/2014 